\title{
Evaluation of Improved and Current Vanillin Based Colorimetric Quantification Methods of Triterpenoids
}

\author{
Rohit Suresh¹, Yash Kamdar², Vivek Garg ${ }^{3+}$, Angelina Kalathoti ${ }^{4+}$, Gayathri \\ Renganathan ${ }^{5^{*}}$ \\ ${ }^{1}$ Lynbrook High School, San Jose, CA, USA \\ ${ }^{2}$ Washington High School, Fremont, CA, USA \\ ${ }^{3}$ Irvington High School, Fremont, CA, USA \\ ${ }^{4}$ Mission San Jose High School, Fremont, CA, USA \\ ${ }^{5}$ Department of Chemistry, Biochemistry, \& Physical Science, Aspiring Scholars Directed Research Pro- \\ gram(ASDRP) Fremont, CA, USA \\ "Corresponding Author:gayathri.renganathan@asdrp.org \\ +denotes 3rd authorship \\ DOI: https://doi.org/10.47611/jsrhs.v10i2.1442
}

\section{$\underline{\text { ABSTRACT }}$}

The most common colorimetric quantification method for triterpenoids utilizes vanillin in strongly acidic conditions to form a colored adduct with the analyte, and it has gained popularity because it is fast and easy to perform. Nevertheless, the detection range of this assay is limited and it is susceptible to side reactions, which cause an interference in the reading. Here, we quantify the rate of this interference in common solvents, and we present an alternative method to minimize the interference, which first incubates the analyte solution in perchloric acid at $60^{\circ} \mathrm{C}$ before the vanillin is added and finally quenched with acetic acid. We also successfully isolated the adduct that formed and confirmed that the color was due to vanillin polymerizing onto the analyte. The improved method had a correlation coefficient of 0.9959 , as well as high accuracy and precision, which had a standard deviation of $0.072 \mathrm{mg} / \mathrm{mL}$, and was not affected by minor changes in the conditions making it a robust method. Furthermore, our method had a limit of quantification of $2.37 \mathrm{mg} / \mathrm{mL}$, which can analyze formulations with ease as well as a limit of detection of 0.782 $\mathrm{mg} / \mathrm{mL}$. Triterpenoids are quickly emerging as bioactive compounds, and as a result, our work improves upon current methods to quantify triterpenoids.

\section{Introduction}

Triterpenoids are natural, bioactive compounds used for their wide spectrum of therapeutic properties ${ }^{1}$. After extracting them from plants, researchers attempt to quantify the total yield, but the most common quantification and detection methods are time consuming and susceptible to inaccuracies. Existing methods for quantification and determination include High Performance Liquid Chromatography (HPLC) and Liquid Chromatography Tandem Mass Spectrometry (LC-MS/MS) $)^{2,3}$. In HPLC, compounds in a sample are separated chromatographically, which is followed with quantification by UV-Vis. Similarly, LC-MS/MS also separates components of a sample, but it ionizes each fraction collected and the mass-charge ratio of the resulting fragments are correlated to analyte concentration. While these methods are quantitative, they suffer from long method development times and high costs per sample 4 . Previous, faster colorimetric detection methods, such as the conventional vanillin assay, result in the formation of a colored vanillinanalyte adduct in acidic conditions, which can be easily quantified with UV-Vis ${ }^{6}$. Despite this, the assay does not account for potential interference from side reactions caused by residual solvents in the sample and concentration of 
the reagents ${ }^{6}$. Therefore, it is important to understand the confounding factors required in detection and quantification because they can limit the practical uses of this assay.

As discussed by Le et al., solvents play a large role in the formation of a colored adduct in the vanillin assay, and others have also reported that running this assay in different solvents create inconsistencies in the data ${ }^{4}$. It was demonstrated that this issue might be circumvented by evaporating the solvent before performing the assay, but this is time consuming and requires high temperatures, which could potentially degrade the analyte ${ }^{7}$. This method has successfully been employed to detect OA in previous studies ${ }^{8}$. Because of the uncertainties surrounding the conventional vanillin method and the methods used by Le et al., we sought to find the optimal solvent for the assay.

We aimed to develop an improved assay in order to accurately quantify triterpenoid concentration. We chose to use oleanolic acid $(\mathrm{OA})$ because of its wide availability and structure. It is a pentacyclic triterpenoid, which means it possesses 5 carbon rings attached together and heteroatoms like oxygen, extracted from Olea europaea, where it is most abundant; however, it is also present in most edible and medicinal plants ${ }^{9,10}$. It has traditionally been used for its medicinal properties such as its anticancer, antimicrobial, antiviral, and anti-inflammatory effects ${ }^{10}$. Moreover, OA shares a common scaffold with many other triterpenoids, several of which are clinically relevant, including ginsenoside, withonolide, ursolic acid, and a-hederin.

Previous studies have investigated how OA behaves in strongly acidic conditions where a carbocation is formed when a carbon atom with four bonds loses two of its valence electrons, resulting in a carbon atom with 3 bonds and a positive charge. As mentioned by Savina et al., OA, the model triterpenoid for quantification, was confirmed to form a carbocation near its alkene by $\mathrm{H} 1 \mathrm{NMR}^{11}$. In addition, OA could potentially undergo lactonization between the carboxyl and the alkene group ${ }^{12}$.

Here, we investigate solvent effects on a vanillin-based colorimetric assay for triterpenoid quantification and screened seven common organic solvents used in extraction processes. Our results suggest that solvents and concentration impact the rate of reaction in the vanillin assay which is relevant for detection and quantification; the differences in solvent effects also provide mechanistic insight into the vanillin colorimetric assay that could be used in future studies on the role of functional groups in the generation of colored adducts.

\section{Methods}

\section{Materials}

A 721 UV-Vis-NIR spectrophotometer was used to record the absorbance at $538 \mathrm{~nm}$. IR spectra were obtained on a Thermo Scientific Nicolet iS5 FT-IR Spectrometer equipped with an iD5 attenuated total reflectance (ATR) sample stage. 1H NMR spectra were collected on a Nanalysis NMReady 60PRO $60 \mathrm{MHz}$ Spectrometer. Unless otherwise stated, all reactions were performed in an Eppendorf tube. Oleanolic acid (OA) was purchased from AK Scientific ( $>95 \%$ purity, used without further purification). All other reagents and solvents were of ACS grade. All experiments were completed in triplicate and subjected to statistical analysis.

\section{Effect of Solvents on Rate}

Because different solvents are composed of different functional groups, studying the rate of color formation in different solvents could provide insight into the mechanism of this assay as well as shed light on its practical implications. $300 \mu \mathrm{l}$ of $8 \% \mathrm{w} / \mathrm{v}$ vanillin in ethanol was added to $300 \mu \mathrm{L}$ of solvents commonly used in natural product extraction, which were 2-butanone, acetone, ethyl acetate (EtOAc), acetic acid ( $\mathrm{AcOH})$, ethanol $(\mathrm{EtOH})$, methanol $(\mathrm{MeOH})$, and isopropanol (Iso). This was mixed thoroughly after $200 \mu \mathrm{L}$ of $70 \% \mathrm{HClO}_{4}$ was added and heated at $60^{\circ} \mathrm{C}$. 


\section{Effect of Concentration of Reactants on Rate}

To determine possible mechanisms of the assay, the amount of acid was kept constant, but the concentration of vanillin in EtOH was doubled and tested in acetone. In another trial, only the amount of acid was doubled. Finally, the effect of doubling both variables was tested.

\section{Isolation of OA-Vanillin Adduct}

To a solution of $20 \mathrm{mg}$ of $\mathrm{OA}$ dissolved in ethanol, $10 \mathrm{~mL}$ of $\mathrm{HClO}_{4}$ was added dropwise at $0^{\circ} \mathrm{C}$ in a round bottom flask. After 15 minutes of heating at $60^{\circ} \mathrm{C}, 200 \mathrm{mg}$ of vanillin was added, and the reaction mixture was stirred and heated for 10 minutes at $60^{\circ} \mathrm{C}$. A TLC in 2:1 EtOAc:hexane was periodically taken, and the complex was isolated with an EtOAc and water extraction. The resulting solution was dried over $\mathrm{MgSO}_{4}$ and concentrated in a vacuum and an IR spectrum of the product was taken.

\section{Improved Assay -- Linearity and Range}

Stock solutions of 5, 4, 3, 2, 1.5, and $1 \mathrm{mg} / \mathrm{mL}$ were prepared by dissolving OA in EtOH. $100 \mu \mathrm{L}$ of the OA solution and $100 \mu \mathrm{L}$ of $\mathrm{HClO}_{4}$ were heated at $60^{\circ} \mathrm{C}$ for 10 minutes. $100 \mu \mathrm{L}$ of $8 \% \mathrm{w} / \mathrm{v}$ vanillin in $\mathrm{AcOH}$ were then added and the solutions were shaken and left for approximately 2 minutes. We performed a standard addition of $1000 \mu \mathrm{L}$ of $\mathrm{AcOH}$ to both quench the reaction and to dilute the sample. $\mathrm{AcOH}$ was chosen to dilute the sample because it preserved the acidity of the solution, which maintained the color. To obtain the calibration curve, we performed linear regression with ordinary least squares minimization, which is a method to minimize the error between the equation of a line and its data points.

\section{Accuracy and Precision}

The accuracy of our improved method was found by performing a standard addition of $30 \mu \mathrm{L}$ of EtOH to $70 \mu \mathrm{L}$ of a $5 \mathrm{mg} / \mathrm{mL}$ sample $(3.5 \mathrm{mg} / \mathrm{mL})$. On the other hand, precision was quantified by independently repeating the trials three times.

\section{$L O D / L O Q$}

The limit of detection (LOD), and this represents the lowest concentration at which the assay will be able to accurately determine if the analyte is present. After plotting the data points from the linear calibration curve, the LOD was calculated with equation 1 , which is the recommended method by the $\mathrm{ICH}$, an organization that provides guidelines on developing analytical methods ${ }^{13}$.

$$
L O D=\frac{3.3 \sigma}{S}
$$

Equation 1. Formula to calculate the LOD. $\sigma$ represents the standard deviation of the y-intercepts of the three lines of best fit while $S$ represents the slope of the line of best fit.

The slope was calculated from the line of best fit from the average of all calibration curve points, and the standard deviation was found from the y-intercepts of the calibration curves from each trial. The limit of quantification (LOQ) is the point where the absorbance can be accurately correlated with concentration. Similar to LOD, the LOQ was calculated as presented in equation 2 .

$$
L O Q=\frac{10 \sigma}{S}
$$

Equation 2. Formula to calculate the LOQ. The terms were substituted with the same values as the LOD equation. 


\section{Robustness}

A robust method will be insensitive to small changes during the execution of the protocol, which commonly occur when multiple people conduct the same method. As a result, we tested this by heating the OA and acid solution between 10 to 25 minutes. Vanillin was then added at each time interval. Furthermore, different vanillin concentrations between 0.08 to $8 \% \mathrm{w} / \mathrm{v}$ were used in the assay to confirm that absorbance was linearly related to vanillin concentration.

\section{Results}

\section{Effect of Solvents on Rate}

Acetone was not heated at $60 \mathrm{C}$ due to its fast reaction rate which would make quantification impossible through UVVis. Because acetone was not heated, the time vs absorbance graph looks close to EtOAc; however, if the acetone reaction were exposed to heat, the graph would be much steeper. 2-butanone followed a rate similar to acetone (without heating), but unlike acetone, which was red, 2-butanone turned green. $\mathrm{EtOH}, \mathrm{MeOH}$, and Iso proceeded at the same rate, but compared to EtOAc, they were approximately 3.5 times slower. All three alcohols as well as EtOAc and $\mathrm{AcOH}$ turned purple (Figure 1a). Cyclohexanone was also tested, but it formed a purple colored product quickly without heating, so quantitative data was not obtained. Furthermore, DMSO was unreactive. Although AcOH has higher absorbances, we still chose to quench the reaction with $\mathrm{AcOH}$ because it preserved the color of the solution which may have occurred because it retained the acidic conditions of the solution.

\section{Effect of Concentration of Reactants on Rate}

When the amount of either vanillin or acid was doubled, the rate also approximately doubled, but when both vanillin and acid were doubled, the rate roughly increased by a factor of 15 (Figure 1b). Despite this, the results can only be generalized to ketones and not the assay in general.

\section{Isolation of OA-Vanillin Adduct}

When the reaction was monitored, one clear spot emerged $(\mathrm{r} f=0.5)$ that was not present in OA-free reactions, but we also noticed a highly polar adduct appearing that streaked the bottom half of the plate. We also saw this product in OA free reactions, so we chose not to isolate it, but it could still contain more polymerized OA-vanillin adducts. The IR spectrum indicated that alcohols, carbonyls, substituted aromatics $(1,2,3,4)$, ethers were present on the compound (Figure 2). In addition, the NMR spectrum did not show the presence of an aldehyde or carboxylic acid, but an aromatic, methoxy, other types of ethers, alkanes, and possibly esters were present (data not shown).

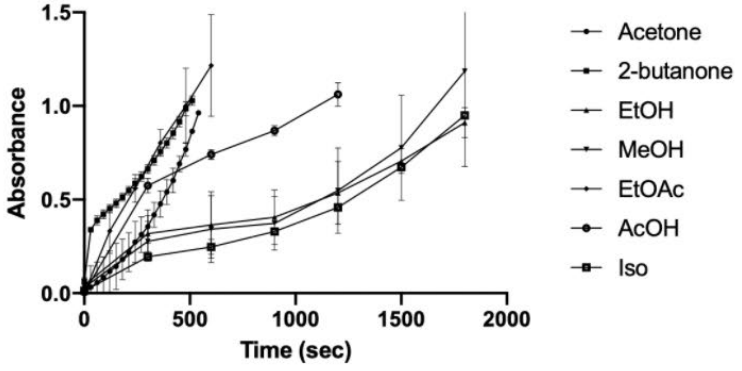

a)

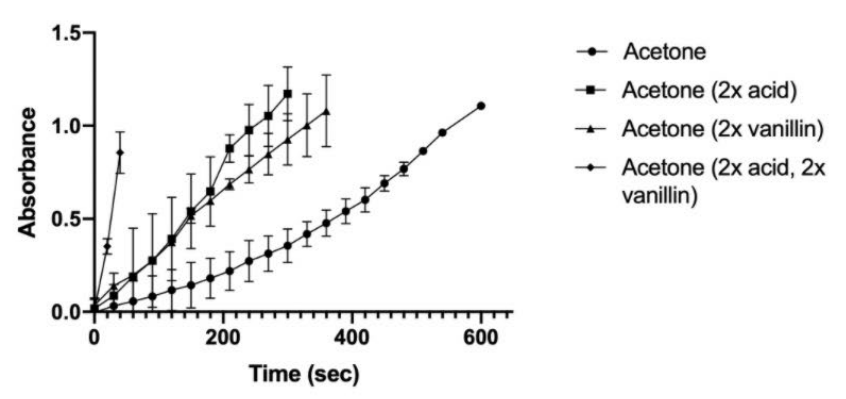

b)

Figure 1. Effect of solvent and concentration of reactants on rate of change of absorbance. 2-butanone and acetone react the fastest, but EtOAc is also relatively fast. In addition, $\mathrm{AcOH}$ has a medium rate while all of the alcohols have the slowest rate (1a). Doubling the amount of vanillin causes the rate to double, and doubling the acid amount causes 
the rate to increase a little bit more than double. Finally, doubling both reactants causes the rate to increase 16-fold (1b).

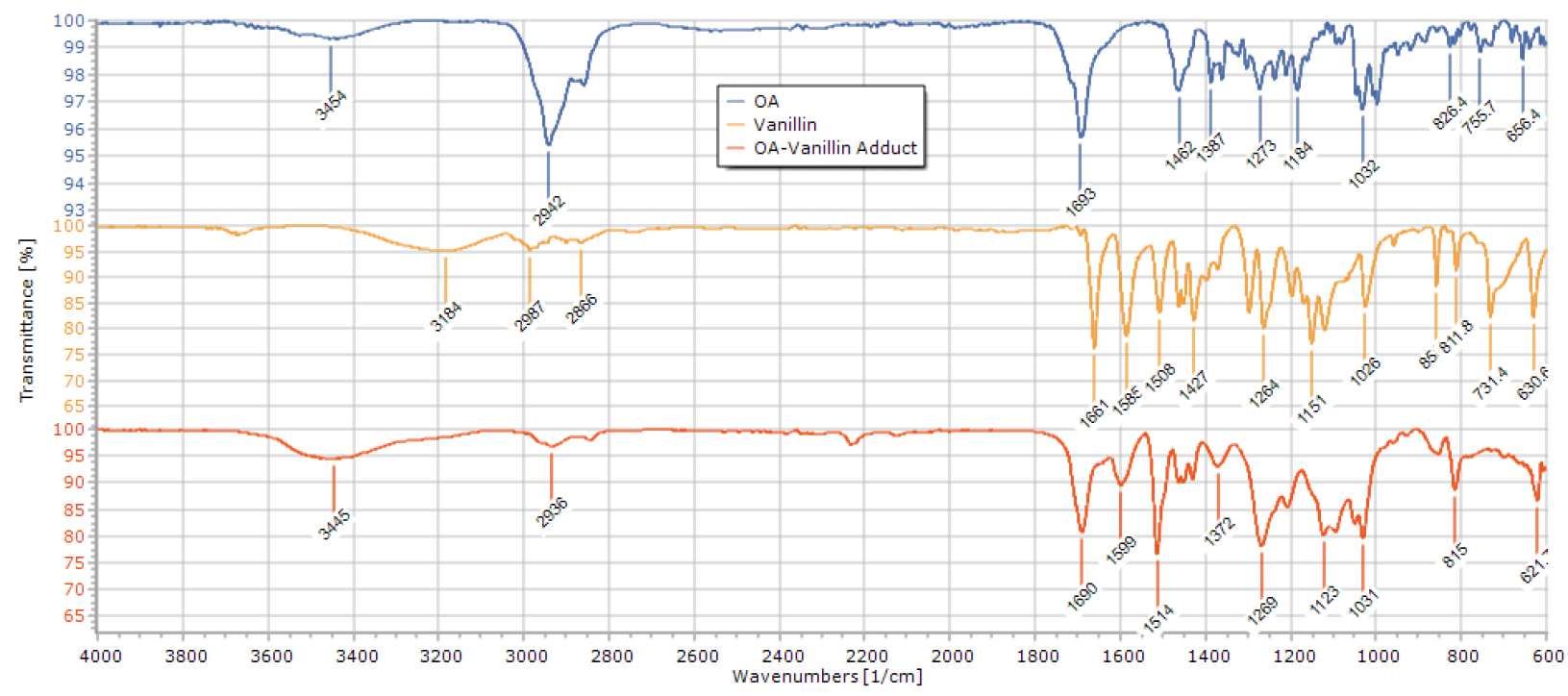

Figure 2. FT-IR spectra of vanillin, OA and the adduct. The carboxyl group is no longer present in the adduct (2942 $\left.\mathrm{cm}^{-1}\right)$. A ketone is present in the adduct while the aldehyde on the vanillin disappeared $\left(1690\right.$ and $\left.1661 \mathrm{~cm}^{-1}\right)$.

\section{Improved Assay — Linearity and Range}

Between the wavelengths of 200 to $800 \mathrm{~nm}$, the OA-Vanillin adduct exhibits strong absorbance at $350 \mathrm{~nm}$, but the smaller peak at approximately $540 \mathrm{~nm}$ is responsible for the solution's purple color (Figure 3). We chose to continue to use $538 \mathrm{~nm}$ as the wavelength for quantification because the signal at $350 \mathrm{~nm}$ could be affected by other compounds in the solution. Of the 6 concentrations tested, only the concentrations between 2 and $5 \mathrm{mg} / \mathrm{mL}$ exhibited linearity (Figure 4A). A linear regression was performed on the linear portion with the ordinary least squares method, and the equation was determined to be $y=0.4069 x-0.45915$ with a strong correlation coefficient $\left(r^{2}\right)$ of 0.9959 (Figure 4B).

\section{Specificity}

Because the assay involves strong conditions, most compounds can react with vanillin to form a colored adduct. As a result, contaminants could significantly affect the accuracy; however, with proper preparation of a blank, an accurate reading can be obtained. The assay is not specific to OA, and it theoretically could be used to quantify samples of other triterpenoids and their respective glycosides. Furthermore, due to the limited solubility of triterpenoids, a pure sample can be easily isolated from a formulation. 


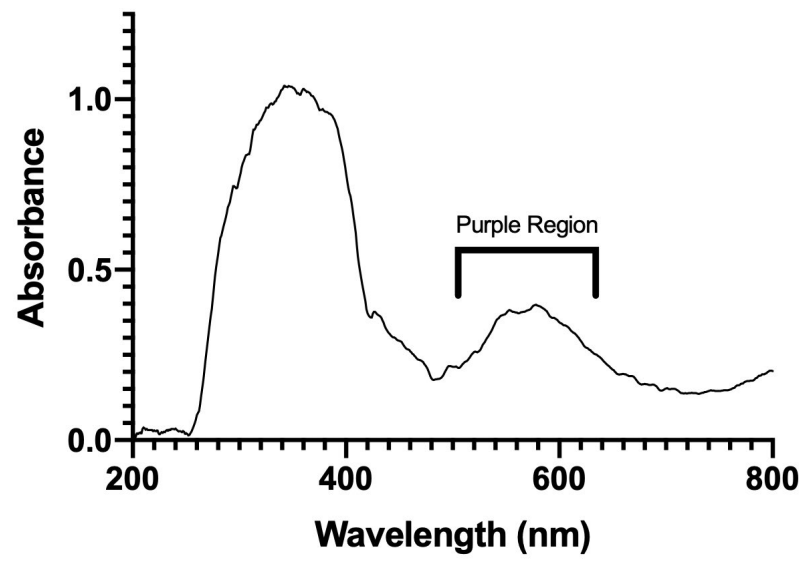

Figure 3. Full absorbance spectrum of the oleanolic acid solution after performing the assay. A large peak is present at $350 \mathrm{~nm}$, but a smaller peak is located at $540 \mathrm{~nm}$, which corresponds to purple.

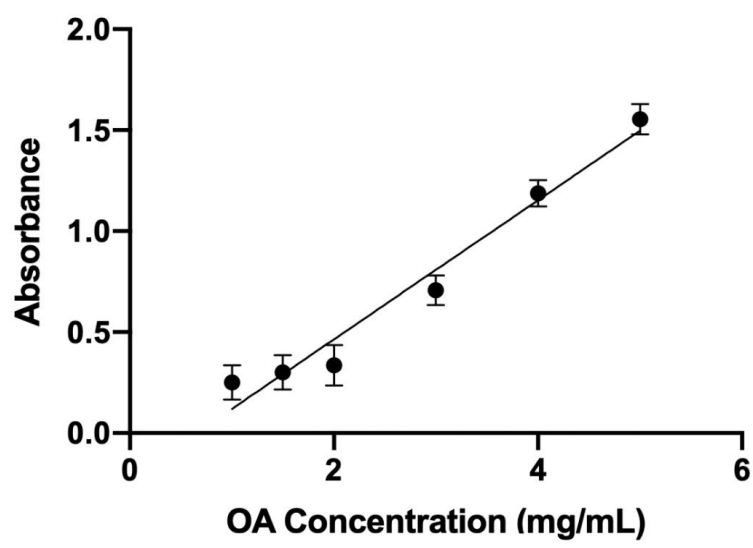

a)

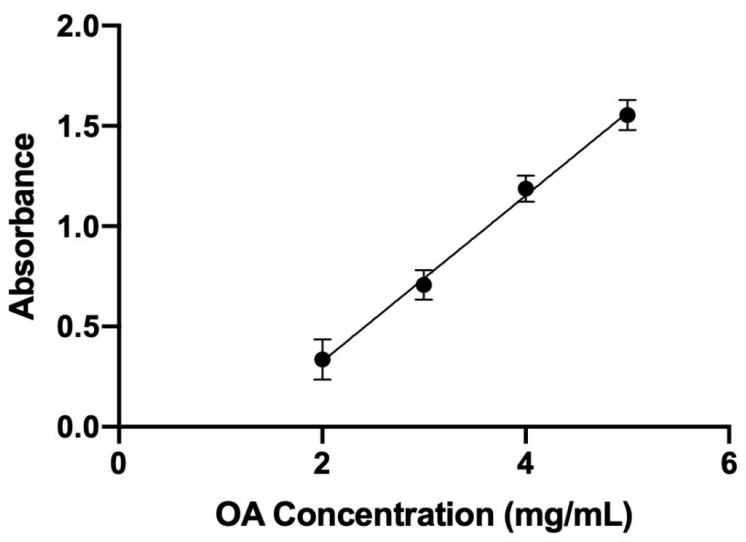

b)

Figure 4. Calibration curve for the improved assay. This method exhibits linearity from $2 \mathrm{mg} / \mathrm{mL}$ to $5 \mathrm{mg} / \mathrm{mL}$ ( $3 \mathrm{~b}$ ) as it has an $r^{2}$ of 0.9959 , but lower concentrations fall below the LOQ (3a), and line has an $r^{2}$ of 0.9587 . Despite this, the assay can still detect these amounts of OA (LOD).

Accuracy and Precision

The method had high precision and a low standard deviation which makes this an ideal assay to routinely quantify samples (Table 1).

\begin{tabular}{|c|c|c|}
\hline Trial & Absorbance & Concentration $(\mathrm{mg} / \mathrm{mL})$ \\
\hline 1 & 0.994 & 3.616 \\
\hline 2 & 0.947 & 3.502 \\
\hline 3 & 0.939 & 3.483 \\
\hline Standard Deviation & 0.030 & 0.072 \\
\hline
\end{tabular}


Table 1. Accuracy and precision of the improved assay. All of the reported values are close together, and they have a standard deviation of $0.072 \mathrm{mg} / \mathrm{mL}$, which is acceptable. In addition, all of the estimated concentrations are close to the expected concentration, which was $3.5 \mathrm{mg} / \mathrm{mL}$.

\section{$L O D / L O Q$}

The LOD was $0.782 \mathrm{mg} / \mathrm{mL}$, and the LOQ was $2.37 \mathrm{mg} / \mathrm{mL}$ over the linear portion of the calibration curve, which is acceptable for the analysis of formulations.

\section{Robustness}

As long as the analyte was incubated in acid for at least 5 minutes, there is little variation in the absorbance of the solution after adding vanillin. There was a standard deviation of 0.048 when the solution was incubated between 10 and 25 minutes (Table 2). This is relevant because it demonstrates that time does not play a large role in the initial phases of this method, which was one of our goals in improving the vanillin method. Separately, when vanillin concentrations between 0.08 to $80 \mathrm{mg} / \mathrm{mL}$ were added to the incubated solution, there was a linear reduction in absorbance. As a result, if samples were to quickly turn black, less vanillin could be added without impacting the accuracy of the method (Figure 5).

\begin{tabular}{|c|c|}
\hline Time (min) in Acid & Absorbance \\
\hline 5 & 1.367 \\
\hline 10 (standard) & 1.587 \\
\hline 15 & 1.476 \\
\hline 20 & 1.513 \\
\hline 25 & 1.553 \\
\hline Standard Deviation & 0.085 \\
\hline Standard Deviation of $10-25$ min & 0.048 \\
\hline
\end{tabular}

Table 2. Effect of time in acid on absorbance of final solution. After 5 minutes of incubation, the time in acid does not change the final absorbance in large amounts. Consequently, small changes in the timing of each sample are unlikely to affect the result. Despite this, it is important to consider that the analyte may degrade if left in acid at high temperatures for too long. 


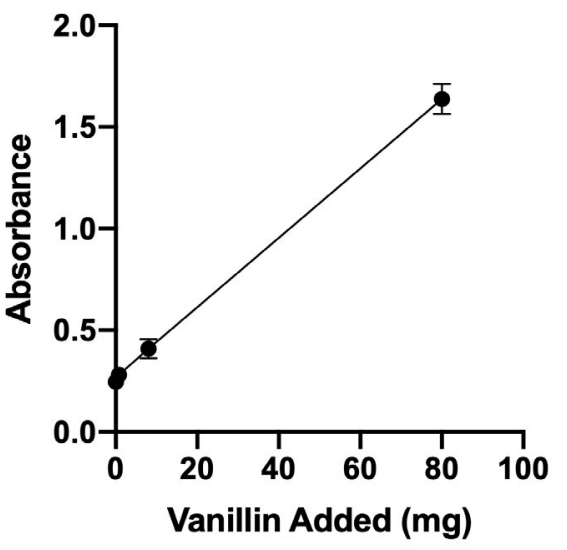

Figure 5. Effect of vanillin concentration on absorbance. The absorbance is proportional to the amount of vanillin.

\section{Discussion}

In general, the presence of a carbonyl group on a solvent increases the rate of the reaction when compared to alcohols. Because this reaction takes place in strongly acidic conditions, this trend in reactivity can be attributed to the rate and stability of carbocation-like characters that forms ${ }^{14}$. Cyclohexanone reacts the fastest because the positive charge can be stabilized with the cyclohexane ring through resonance. 2-butanone reacts faster than acetone because it has 4 linear carbons available to stabilize it. Surprisingly, all three of the alcohols tested had the same reaction rates and absorbance, which suggests a similar reaction mechanism such as a substitution on the alcohol that does not depend on substituents. The hydroxyl on $\mathrm{AcOH}$ is also able to act as a good leaving group when protonated. On the other hand, EtOAc is capable of undergoing keto-enol tautomerism, which could provide it with nucleophilic properties; thus, allowing it to react with vanillin. Although it is unexplained why DMSO is unreactive, this could lead to low background noise levels, so it could be used as a solvent for this assay. Figure 6 shows the different mechanistic pathways on alcohols and ketones.

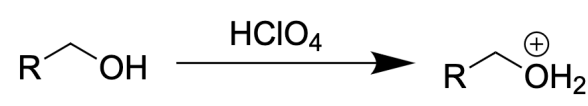

a)

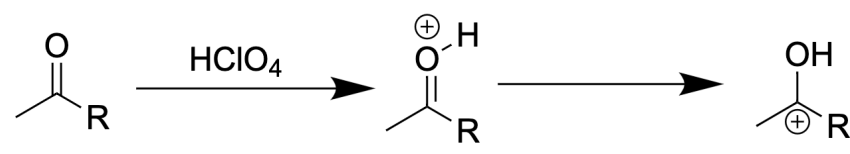

b)

Figure 6. Mechanism of reactivity on alcohols and ketones. In figure 6a, the alcohol becomes protonated under the strong acid. The protonated alcohol is capable of acting as a good leaving group in substitution reactions. Similarly in figure $6 \mathrm{~b}$, ketones follow a similar pathway, but the alcohol remains; as a result, the positive charge is resonance stabilized between the carbon and protonated ketone.

According to the above mechanism, the ketone solvents retain the $\mathrm{C}-\mathrm{O}$ bond during the reaction with vanillin, so more exotic colors could form such as red and green in acetone and 2-butanone respectively.

Although doubling one reactant at a time showed a change in rate somewhat consistent with first-order reactions, doubling both reactants increased the rate by $12-16$ fold, which is not consistent with first-order reactions, and as a 
result, a more complex reaction mechanism must exist. This signifies that vanillin and perchloric acid play large roles in both the consistency and result of the assay.

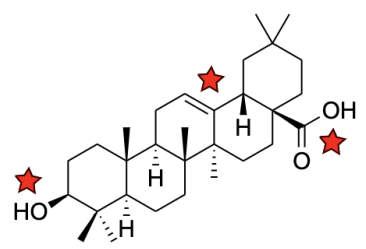

Figure 7. Possible sites of protonation on OA. The stars indicate the areas where OA is capable of becoming protonated. The alkene, which is the most reactive group on OA, can become a carbocation, and it is the most stable because it is stabilized through the ring system. The $\mathrm{OH}$ on the carboxyl group can become protonated to become a leaving group, and the secondary alcohol can also do this. In addition, the secondary alcohol could also become dehydrated to form a carbocation. Similar mechanisms to solvents are present for carbocation formation on the secondary alcohol and carboxylic acid of OA.

OA likely reacts at the alkene, carboxyl, and alcohol sites because those functional groups can easily form carbocations, which are carbons with a positive charge, or good leaving groups. A leaving group is a functional group that can be replaced by another reactant relatively easily during the reaction. Because we ran the assay in EtOH, the alkene may have reacted with the solvent to form an ether. Another possibility could involve the carboxyl group reacting with the alkene to cyclize ${ }^{12}$. The assay produces a purple adduct, which is similar to the color of both acetic acid and ethanol, so the alcohol and carboxyl sites are the most likely to be reacting with the vanillin (Figure 7). When the IR of the adduct is compared to an IR of pure OA, the alkene and alcohol peaks disappear, which confirms the reactivity at those sites ${ }^{15}$. Furthermore, the IR confirms the carboxyl reacts, but it is unknown what it reacted with. The OA was dissolved in ethanol, and if the assay were performed as previously reported, moderate amounts of background noise could be created, which could interfere with the accuracy of the method. Adding the acid first prevents this by quickly protonating the OA but not affecting the ethanol. The resulting solution is orangish-pink. When the vanillin is added, the absorbance proportionally increases, which indicates a polymerization must be occurring.

Vanillin has been proven to possess both nucleophilic and electrophilic properties, but because positively charged groups are forming on OA, an electrophile, vanillin must be acting as the nucleophile, which reacts with the electrophile, in this assay ${ }^{16}$. The vanillin has two activating groups on the benzene ring, which are the hydroxyl and methoxy groups. They both donate lone pairs into the ring, which become stabilized by resonance. As a result, the benzene ring of vanillin is electron-rich, providing it with nucleophilic properties. Through an electrophilic substitution reaction, the vanillin reacts with the electrophile on OA to form a bond. The electron is then transferred to the alcohol making a protonated ketone, which can then react with another vanillin to create a polymer (Figure 6). The two peaks of absorbance in the full UV-Vis spectrum can be attributed to possible resonance structures or the adduct could possess two separate peaks. Carbocation radical polymerization has previously been reported with aromatics, and a similar mechanism could be occurring in this assay ${ }^{17}$. This proposed mechanism is consistent with the NMR data obtained as a doublet at 7.00, which represents the hydrogens on C-7, is missing in the OA-vanillin product (data not shown). Theoretically, the substitutions could occur in both the ortho and meta positions on vanillin, which cover all positions on the benzene ring besides the carbon directly opposite the aldehyde, but the NMR data suggests that the meta positions remain unreactive because the doublet at 7.48 remains in the adduct (data not shown). It is also unclear if the aldehyde participates in the polymerization or if it reacts with the solvent to form acetals or hemiacetals, which would confirm the alcohol present in the IR. To simply put it, the vanillin acts as a color developing agent (Figure 8). 
<smiles>COc1cc(C=O)ccc1O</smiles>
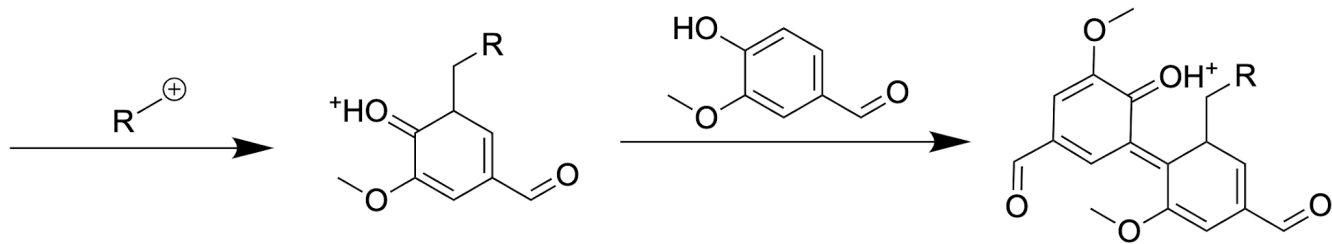

Figure 8. Polymerization of vanillin after reacting with an initiator molecule. The initiator molecule undergoes an electrophilic substitution on the benzene ring, and this causes a radical to form another leaving group on the phenol. The phenol then undergoes an electrophilic substitution again with another vanillin to polymerize. The aldehydes could react to form hemiacetals or acetals.

In theory, any compound that could easily form carbocation-like characters such as ketones could interfere with the assay. Despite this, the improved assay presented in this work is more accurate than previously reported versions of the vanillin assay through a wider range of concentrations, which will become relevant in the coming years as triterpenoids gain traction as natural products with medicinal value. Future research is being performed to isolate and characterize the second adduct through LC-MS/MS. Further research should focus on lowering the LOQ and fully elucidating the reaction mechanism of colorimetric vanillin assays.

\section{Conclusion}

Herin, we have revealed that previously reported versions of the vanillin assay do not address the effects of solvent, which could significantly impact the accuracy and precision of the method due to side reactions leading to increased colored adduct formation. The implications of this work identify the solvents that future researchers should consider using during natural product isolation, which they plan to quantify with the vanillin assay. Despite this, some extractions require a specific set of solvents, and one workaround is to evaporate the solvent then add a suitable solvent, like DMSO or ethanol, to dissolve the analyte. Furthermore, we have presented an alternative solution that aims to improve the accuracy and precision as well as equip the assay to be able to quantify larger amounts of compound, which the previous vanillin assay was unable to do. Our method demonstrated an acceptable LOD and LOQ as well as high robustness. We have confirmed that the assay proceeds through a complex mechanism through reaction rate analysis and involves polymerization, which proportionally increases color intensity. In addition, a proposed mechanism for vanillin polymerization in highly acidic conditions was hypothesized as well its connection to the detection of triterpenoids. Although our proposed mechanism matches with the IR spectrum obtained, we were able to identify multiple polymerization products appearing during the assay; as a result, future studies should attempt to isolate polymerized products separately and characterize the adduct through alternative methods. We have successfully demonstrated that with some adjustments to the assay parameters based on a proposed mechanism of the assay, a more valid and reliable assay can be used for analytical purposes in a wide range of fields.

\section{Acknowledgments}

RS and GR conceptualized the project, and RS and YK wrote the manuscript, developed, and troubleshooted the methods. VG and AK assisted with lab work. In addition, we are grateful for our advisors' support throughout this project by providing us with ideas to further our work. We would like to thank the Aspiring Scholars Directed Research Program (ASDRP) in Fremont, CA for supporting us with the facilities, instrumentation, and funding to conduct this research. We appreciate the lab faculty for providing us with technical assistance to conduct our experimentation. Furthermore, we gratefully acknowledge Edward Njoo for providing help with the analytical analysis in this work and providing us with suggestions on our manuscript. 


\section{References}

[1] Wu, G., Guo, J., Bao, J., Li, X., Chen, X., Lu, J., \& Wang, Y. (2013). Anti-cancer properties of triterpenoids isolated from Ganoderma lucidum - a review. Expert Opinion on Investigational Drugs, 22(8), 981-92.

[2] Taddeo, V., Castillo, U., Martínez, M., Menjivar, J., Jiménez, I., Núñez, M., \& Bazzocchi, I. (2019). Development and Validation of an HPLC-PDA Method for Biologically Active Quinonemethide Triterpenoids Isolated from Maytenus chiapensis. Medicines, 6(1), 36.

[3] Zhu, D., Zhou, Q., Li, H., Li, S., Dong, Z., Li, D., \& Zhang, W. (2018). Pharmacokinetic Characteristics of Steamed Notoginseng by an Efficient LC-MS/MS Method for Simultaneously Quantifying Twenty-three Triterpenoids. Journal of Agricultural and Food Chemistry, 66(30), 8187-98.

[4] Cheok, C.Y., Salman, H.A.K., \& Sulaiman, R. (2014). Extraction and quantification of saponins: A review. Food Research International, 59, 16-40.

[5] Wang, Y., \& Yang, Y. (2007). Simultaneous quantification of flavonoids and triterpenoids in licorice using HPLC. Journal of Chromatography B, 850(1-2), 392-9.

[6] Hiai, S., Oura, H., \& Nakajima, T. (1976). Color Reaction Of Some Sapogenins And Saponins With Vanillin And Sulfuric Acid. Planta Medica, 29(02), 116-22.

[7] V. Le, A., E. Parks, S., H. Nguyen, M., \& D. Roach, P. (2018). Improving the Vanillin-Sulphuric Acid Method for Quantifying Total Saponins. Technologies, 6(3), 84.

[8] Xiang Zhaobao, Tang Chunhong, Chen Gang, Shi Zhisong. Studied on corlorimetric determination of oleanolic acid in Chinese quince Natural Product Research and Development. 2001 ;13(4):23-26.

[9] Zhou, Q., Du, G. (2018). Oleanolic Acid. Natural Small Molecule Drugs from Plants, 1, 433-437.

[10] Ayeleso, T., \& Matumba, M. (2017). Oleanolic Acid and Its Derivatives: Biological Activities and Therapeutic Potential in Chronic Diseases. Molecules, 22(11), 1915.

[11] Savina, A. A., Fesenko, D. A., Sheichenko, V. I., \& Shavlinskii, A. N. (1986). Nature of the transformations of oleanolic acid in an acid medium. Chemistry of Natural Compounds, 22(3), 295-297. doi:10.1007/bf00598299

[12] Oganesyan, É. T. (1980). Mechanism of the reaction of triterpenoids with sulfuric acid. Chemistry of Natural Compounds, 16(5), 464-468. doi:10.1007/bf00571039

[13] ICH Harmonized Tripartite Guidelines (2005) Validation of analytical procedures: Text and methodology, Q2 (R)

[14] Arnett, E.M., \& Hofelich, T.C. (1983). Stabilities of carbocations in solution. 14. An extended thermochemical scale of carbocation stabilities in a common superacid. Journal of the American Chemical Society, 105(9), 2889-95. [15] Seebacher, W., Simic, N., Weis, R., Saf, R., \& Kunert, O. (2003). Complete assignments of1H and13C NMR resonances of oleanolic acid, 18?-oleanolic acid, ursolic acid and their 11-oxo derivatives. Magnetic Resonance in Chemistry, 41(8), 636-8.

[16] Semenov, S.G., \& Makarova, M.V. (2010). Quantum-chemical study of the molecular forms of vanillin. Russian Journal of General Chemistry, 80(9), 1806-11.

[17] GOETHALS, E., \& DUPREZ, F. (2007). Carbocationic polymerizations. Progress in Polymer Science, 32(2), $220-46$. 